\title{
El componente cultural en los diccionarios de ELE - análisis de los artículos gazpacho, sangría, bocadillo, paella, albergue y posada
}

The cultural component in dictionaries of SFL - analyses of the terms gazpacho, sangría, bocadillo, paella, albergue and posada

\begin{tabular}{|c|c|}
\hline $\begin{array}{l}\text { RESUMEN: El diccionario es una } \\
\text { herramienta didáctica que busca } \\
\text { incorporar los aspectos lingüísticos y } \\
\text { extralingüísticos de una lengua (PORTO } \\
\text { DAPENA, 2014). Entre estos aspectos, } \\
\text { cabe mencionar los relacionados con las } \\
\text { costumbres, la historia, las curiosidades y } \\
\text { la cultura de una sociedad (ATIENZA } \\
\text { CEREZO, 2005; BLECUA PERDICES, } \\
\text { 2016). Podemos señalar que el diccionario } \\
\text { es una obra pedagógica que debe recoger } \\
\text { este tipo de información necesaria para } \\
\text { los consultantes, sobre todo los dirigidos } \\
\text { a la enseñanza y aprendizaje de los } \\
\text { usuarios extranjeros según sus } \\
\text { necesidades (MALDONADO, 2008). En } \\
\text { este sentido, este estudio se centra en } \\
\text { analizar seis artículos del ámbito del } \\
\text { Turismo extraídos del Diccionario para la } \\
\text { Enseñanza de la Lengua Española para } \\
\text { Brasileños (SENAS, 2003), obra } \\
\text { lexicográfica de gran difusión en la } \\
\text { enseñanza de ELE en Brasil (MOREIRA, } \\
\text { 2013; SIMÃO, 2016) y del Diccionario de } \\
\text { Español para Extranjeros. Describimos las } \\
\text { definiciones y los ejemplos de uso de las } \\
\text { entradas con el fin de averiguar si } \\
\text { resultan o no informativas, con el }\end{array}$ & $\begin{array}{l}\text { ABSTRACT: Dictionary is a didactic tool } \\
\text { that seeks to incorporate the linguistic } \\
\text { and extra linguistic aspects of a language } \\
\text { (PORTO DAPENA, 2014). Among these } \\
\text { aspects, it should be mentioned those } \\
\text { related to the customs, history, curiosities } \\
\text { and culture of a society (ATIENZA } \\
\text { CEREZO, 2005; BLECUA PERDICES, } \\
\text { 2016). We can say that a dictionary is a } \\
\text { pedagogical tool that should mark this } \\
\text { type of information necessary for the } \\
\text { consultants, especially those directed to } \\
\text { the teaching and learning of foreign users } \\
\text { according to their needs (Maldonado, } \\
\text { 2008). In this sense, this study focuses on } \\
\text { analyzing six entries from the scope of } \\
\text { Tourism extracted from Diccionario para la } \\
\text { Enseñanza de la Lengua Española para } \\
\text { Brasileños (SEÑAS, 2003), a lexicographic } \\
\text { work of great diffusion in the teaching of } \\
\text { ELE in Brazil (MOREIRA, 2013; SIMÃO, } \\
\text { 2016) and the Diccionario de Español para } \\
\text { Extranjeros. We described the definitions } \\
\text { and examples of use of the entries in order } \\
\text { to find out whether they are informative } \\
\text { or not, and if it is necessary to include } \\
\text { other type of information and which one. } \\
\text { The results show that it is imperative to }\end{array}$ \\
\hline
\end{tabular}

* Doctorando en Traducción y Ciencias del Lenguaje en la Universitat Pompeu Fabra (UPF-IULA). Profesor de Español en la Universidade Federal do Piauí(UFPI). glauberlimamoreira@gmail.com 
propósito de averiguar si es necesario añadir otros tipos de informaciones y cuáles. Los resultados muestran que es imprescindible elaborar un diccionario de ELE pensado para los usuarios brasileños y que las informaciones enciclopédicas y culturales son fundamentales para este público.

PALABRAS CLAVE: Enseñanza de ELE. Componente cultural. Diccionario. Microestructura. Turismo. elaborate an IT dictionary designed to Brazilian users and that encyclopedic and culture information is fundamental for this audience.

\section{Palabras iniciales}

El interés, la motivación y las diferentes necesidades de un individuo, en un contexto educacional, profesional o no, en la búsqueda para aprender y/o aprehender un idioma extranjero ( $\mathrm{y}$ también materno) se debe a diversos motivos, sean cuestiones de índole profesional, educativo, ampliación del léxico del idioma meta o simplemente por el mero placer e interés personal de acercarse a un nuevo universo lingüístico y cultural; o también para la ampliación del léxico en el idioma de llegada.

Una de las ramas de la ciencia lingüística que se ocupa de investigar la(s) metodología(s) apropiada(s) y eficiente(s) para aplicar en el aula y para obtener el adecuado desarrollo de los contenidos estudiados durante el proceso de la enseñanza y del aprendizaje de la lengua extranjera (en adelante LE) es la Didáctica de las Lenguas, la cual las teorías que comprenden esta ciencia muestran y comprueban que existen diferentes métodos y enfoques pedagógicos que deben ser aplicados en clase, dependiendo del nivel lingüístico y del perfil del estudiante, como también los objetivos de aprendizaje que el alumnado desea alcanzar, con el fin de profundizar y retener los contenidos lingüísticos, pragmáticos y culturales relacionados con el código que está empezando a apropiarse gradualmente el aprendiente. 
En este sentido, diversas investigaciones como, por ejemplo, Ahumada (2006), Rodríguez Barcia (2016), Biderman (1998) y Maldonado (2012) señalan que el diccionario es un buen ejemplo de material pragmático vivo del que el estudiante, sobre todo el extranjero, en el caso del presente estudio el brasileño, puede obtener innumerables beneficios de los conocimientos marcados en él, los cuales son necesarios para el desarrollo eficaz de su aprendizaje en la LE, sean sintácticos, semánticos e, incluso, sociopragmáticos, llamados también de contenidos culturales, siendo la principal finalidad de este estudio.

De hecho, el repertorio lexicográfico es considerado por varios investigadores (BATTANER, 2014; SVÉNSEN, 2009; PORTO DAPENA, 2014) como una herramienta didáctica que busca registrar los aspectos lingüísticos y extralingüísticos de una lengua, sea materna o extranjera, tanto los de tipo bilingüe como monolingüe, como también los semibilingües, clasificados también como bilingualizados. Entre estos aspectos, cabe mencionar los relacionados con las costumbres y la cultura de una sociedad determinada (ATIENZA CEREZO, 2005; BLECUA PERDICES, 2016), estimamos que la inclusión de estas cuestiones es esencial en el cuerpo del diccionario para que el aprendiz extranjero (re)conozca las pueda reconocer, lo que constituye el foco principal de este estudio, pero no únicamente.

El diccionario, como hemos señalado anteriormente, es una obra, didáctica, pedagógica o de otro tipo, que puede y debe recoger este tipo de informaciones necesarias e indispensables para la competencia de los consultantes brasileños, independiente de su nivel lingüístico, sobre todo en los repertorios lexicográficos dirigidos especialmente para la enseñanza y aprendizaje de los usuarios extranjeros, tomando en consideración sus necesidades (MALDONADO, 2008; MALDONADO; NADIN, 2017). Somos plenamente conscientes de que el diccionario no es una enciclopedia, pero defendemos que en él existe espacio suficiente y apropiado para 
incluir informaciones de este tipo tanto en la parte de la definición como en los ejemplos de uso (GARRIGA ESCRIBANO, 2001; REY-DEBOVE, 2005).

Por tanto, el objetivo principal del presente trabajo es analizar y demostrar, de manera sucinta pero lo más contundente posible que el diccionario no registra, en la mayoría de las ocasiones, o lo expone de manera superficial, el componente cultural en su microestructura y, más específicamente, en los paradigmas lexicográficos referentes a la definición y a los ejemplos de uso, en el Diccionario para la Enseñanza de la Lengua Española para Brasileños (SEÑAS, 2001), obra lexicográfica de gran difusión y empleo en la enseñanza de español como lengua extranjera (en adelante ELE) en Brasil (MOREIRA, 2013, 2017; SIMÃO, 2016), y por esta razón elegida como objeto de análisis para este trabajo.

Este artículo está organizado de la siguiente manera: la primera parte se dedica a las consideraciones teóricas pertinentes al estudio, las cuales están compuestas por las principales ideas referentes a la definición lexicográfica y los ejemplos de uso en los diccionarios y sobre la enseñanza del léxico de un idioma para fines específicos, concretamente a la enseñanza de ELE para el Turismo. La segunda parte presenta la metodología del estudio. Finalmente, en la tercera, el análisis de los artículos lexicográficos, discutimos el tratamiento que reciben las definiciones lexicográficas y los ejemplos en los repertorios seleccionados.

\section{La lexicografía pedagógica y el uso del diccionario en el aula}

La lexicografía pedagógica es una de las ramas de la Lexicografía, la ciencia de los diccionarios. Esta disciplina científica es responsable, sobre todo, de investigar, dentro del universo de las temáticas de estudio, cómo, cuándo y para qué los usuarios utilizan el diccionario, además de saber si son obras que sirven, adecuada y eficazmente, para diseminar el aprendizaje del individuo, independientemente de la asignatura en la que se emplea y de la lengua utilizada. La lexicografía didáctica, por 
ende, tiene el objetivo de fomentar la utilización de las obras lexicográficas, en el aula y fuera de ella, como un recurso plurilingüístico que ofrece al usuario un abanico de informaciones que, muchas de las veces, lamentablemente, el consultante no toma en consideración, desechando la idea de que en ellas podrá encontrar otra clase de contenidos, puesto que muchos de ellos consultan los diccionarios para saber simplemente cómo se escribe una determinada palabra, su significado y cómo se dice la palabra $x$ en una dada lengua (MARTÍNEZ EZQUERRO, 2001; MALDONADO; NADIN, 2017).

No obstante, los investigadores Lehman y Martin-Berthet, (2013) y Domínguez Vázquez y Valcárcel Riveiro, (2015), por mencionar algunos, afirman que se debe usar frecuentemente el diccionario y en diferentes contextos y motivos, pero, para que ello se concretice adecuadamente sin comprometer el aprendizaje, es imprescindible que el usuario (re)conozca muy bien la estructura de esta obra y sepa el provecho que puede obtener de dicha herramienta, dado que, debido al poco conocimiento acerca de los diccionarios, esta actitud restringe su uso, sin considerarlo un instrumento coadyuvante en el proceso de la enseñanza-aprendizaje de significativa relevancia.

El Marco Europeo Común: de referencia para las lenguas: aprendizaje, enseñanza, evaluación (2002: 41), constatando la importancia del uso de los recursos didácticos de tipo lexicográficos stricto sensu, en especial, los diccionarios, en el aula de ELE, afirma claramente la importancia del diccionario para el usuario, al decir que "Con la ayuda de un diccionario, puede comprender mensajes escritos de forma sencilla, y sin diccionario, puede comprender lo esencial."

De la misma forma que Aragonés (2001) señala sobre la importancia de la utilización del diccionario en el ámbito escolar, acreditamos que dicho manual didáctico es una herramienta que funciona, o debería, como un elemento metodológico, motivador y práctico, indispensable para el desenvolvimiento tanto de la comprensión lectora como de la producción textual del aprendiz de LE, además de 
las otras destrezas. Como afirma, aun, Aragonés (2001, p. 209), parece evidente que el diccionario es un complemento imprescindible para el aprendizaje y también para el dominio de la lengua, cuyo uso habitual por parte del consultante puede ayudarlo a mejorar el conocimiento del código lingüístico y, especialmente, a ampliar su caudal lexical y a utilizar el lexicón que él ya posea con más precisión, propiedad y seguridad. En este sentido, para el autor brasileño Pontes (2008, p. 29): “O dicionário é um texto e, como tal, permite leituras. Serve de ferramentas para a construção de outros textos".

Sin embargo, para que el repertorio lexicográfico se convierta en un instrumento útil para los fines señalados arriba, estamos de acuerdo con la investigadora Torre Madueño (2001, p. 282) al afirmar que un grupo de docentes preparado en la enseñanza de una lengua específica con una adecuada formación pedagógica (y lexicográfica) para la utilización de su material pedagógico contribuirá, sin lugar a dudas, a una mayor eficacia en lo relativo a su práctica didáctico-metodológica referente al uso de este material. Mientras que un cuerpo de discentes concentrado y motivado en adquirir información a partir de las fuentes lexicográficas fomentará una mayor capacidad y dedicación por parte del profesor, cuyos beneficios repercuten directamente, obviamente, en el conocimiento de los alumnos, extranjeros o no, sobre la lengua meta.

Alvar Ezquerra (2003, p. 13), a este respecto, plantea la siguiente información sobre el conocimiento necesario del grupo de profesorado acerca del diccionario como más una herramienta de significativa utilidad en el desarrollo del aprendizaje del estudiante de ELE:

El empleo que hagan del diccionario los estudiantes durante el aprendizaje de la lengua dependerá de las instrucciones que le dicte su profesor. Por eso es por lo que resulta importante y necesario que el profesor conozca los diccionarios y sepa qué partido puede sacar de ellos. (la cursiva es nuestra). 
En esta misma línea de pensamiento, Hernández Hernández (1989) afirma que los docentes que quieren y necesitan obtener un significativo éxito en su trabajo pedagógico y laboral, es decir, que posibiliten mejorías concretas a su práctica docente en lo que concierne a sus actividades realizadas en el aula, necesitan del (de los) conocimiento(s) necesario(s) sobre la estructura, la función y los contenidos disponibles en las obras lexicográficas, además de la destreza de estrategias de búsqueda de los referidos libros.

Esta afirmación coindice con el pensamiento expresado por Ávila Martín (2000, p. 30) quien indica manifiestamente que "[...] el diccionario responde a necesidades de información y comunicación, es didáctico; es un texto finito, un producto, una praxis en la que se hallan implicados el autor y el lector [...]." Dicho de otra manera, el diccionario es un libro que nosotros, los usuarios, necesitamos conocer su estructura y función con precisión y, con ello, profesores y aprendices brasileños de ELE, pueden y deben, sin ningún recelo, utilizar con bastante provecho la gama de informaciones que el diccionario ofrece a sus lectores-consultantes.

Asimismo, de manera general, es un hecho confirmado en varias investigaciones realizadas, como por ejemplo en la de Araújo (2007), que una gran parte del profesorado del ámbito de la enseñanza de una LE ha experimentado situaciones en las que el diccionario transmite una explicación que parece insatisfactoria a su alumnado sobre el significado de una determinada palabra, porque el diccionario del estudiante decía algo diferente al suyo, es decir, no correspondía a las expectativas y tampoco aclaraba de todo las dudas del aprendiz, pues el usuario no es consciente de que no todos los diccionarios sirven para realizar las búsquedas de los usuarios en cualquier momento (SANMARTÍN SAÉZ, 2017), por lo que la referida situación provoca un descontento en el usuario, puesto que no presenta suficiente información sobre las voces objeto de su interés. 
Esta realidad, que experimentan muchos profesores de enseñanza de ELE de no saber elegir la obra y explorar los conocimientos que el diccionario ofrece para impulsar el proceso de aprendizaje de una lengua, nos muestra el desconocimiento de los docentes de estas herramientas lexicográficas. Está claro que las obras lexicográficas presentan limitaciones y virtudes, pues la inmensa diversidad de una lengua, su dinamismo, sus innumerables transformaciones lingüísticas, sobre todo, la lengua hablada en la calle, etc., son algunas de las informaciones de tipo cultural que no tienen cabida, usualmente, en un diccionario; no obstante, las consideramos indispensables para el aprendizaje de un escolar extranjero y, por tanto, defendemos que los hacedores de diccionarios deben marcarlas en la microestructura lexicográfica, ofreciendo, de este modo, más informaciones extralingüísticas al lector no nativo. En este sentido, lo expuesto anteriormente confirma la teoría defendida por la autora Krieger (2005, p. 12) al decir que: “Apesar do reconhecimento unânime de suas funções didáticas, este tipo de obra é ainda um objeto bastante desconhecido e mesmo pouco explorado [...]", por parte de sus diferentes usuarios y lectores. Por esta razón, nosotros, profesores y estudiantes, no acudimos a menudo está herramienta, y es importante decir que tal declaración coincide en la entrevista de Maldonado y Nadin (2017).

En consecuencia de lo presentado hasta aquí, podemos afirmar que las investigaciones muestran que los profesores no suelen estar adecuadamente instrumentados/instruidos para realizar un trabajo más sistemático, adecuado y apropiado con los diccionarios para su uso en las clases, sean de lenguas o no; y, consecuentemente, ni se sienten seguros al elegir una clase de obra para manejarla en el contexto escolar; ni tampoco cómodos para sugerir a los aprendientes una determinada obra, porque no han recibido una preparación teórico-práctica apropiada y suficientemente capacitada y que, muchas veces nula, durante su formación académica universitaria, momento que consideramos crucial para conocer y aprender 
a manejar correctamente para sacar y aprovechar el máximo provecho de estas obras (EGIDO VICENTE, 2017; GONZÁLEZ, 2017; AZORÍN FERNÁNDEZ; SANTAMARÍA PÉREZ, 2017).

Así pues, podemos llegar a la comprensión de que el conocimiento para utilizar la obra lexicográfica no se adquiere por sí mismo, es decir, de manera irreflexiva, de forma aleatoria y sin conocimientos formales. Por eso, es necesario que el docente esté preparado, teóricamente hablando, para el empleo de esta obra en el aula de LE, y pueda colaborar efectivamente con el desempeño escolar de sus discentes, futuros usuarios en potencia, para que, al final, todos los sujetos involucrados empleen adecuadamente esta herramienta de apoyo para el desarrollo eficiente de la comunicación de la lengua objeto en cuestión.

\section{La enseñanza de ELE y la incorporación de los contenidos culturales en el diccionario}

En el ámbito de las teorías presentadas por Biderman (1998, p. 129), esta investigadora afirma que solo el diccionario general de la lengua puede acercarse al ideal de describir y documentar el léxico de esta lengua. Sin embargo, este ideal es siempre inalcanzable, ya que el léxico crece en progresión geométrica, actualmente, sobre todo, en virtud de la gran aceleración de los cambios socioculturales y tecnológicos. En rigor, ningún diccionario, por más voluminoso que sea, dará cuenta integral de marcar el léxico de una lengua de civilización (la cursiva es nuestra). Esto también sucede con los diccionarios de aprendizaje, por lo que los contenidos de este tipo no deben ser ignorados por los responsables que elaboran estas herramientas.

No obstante, con los cambios que la lengua confronta, estamos seguros de que las informaciones culturales deben, indudablemente, ser contempladas en los artículos lexicográficos, en formato impreso y en línea, para que, de alguna manera, el lector aprenda la manera de vivir, actuar, reaccionar, ver y explicar el mundo de la comunidad de los hablantes de la LE que esté estudiando, pues dichas informaciones 
pragmáticas ayudan al proceso de la comunicación en otra lengua con más elocuencia (ATIENZA CEREZO, 2005, p. 8; TARP, 2008).

Es importante señalar que las conclusiones de muchos estudios que buscaron saber, por ejemplo, la frecuencia de uso del diccionario por parte de los estudiantes muestran que estos sujetos no encuentran mucho sentido a realizar consultas con frecuencia, ya que no solo quieren conocer una información más concreta como, por ejemplo, si el lema límite lleva o no tilde y por qué, cómo se separa y escribe la palabra perro o cómo se dice sándwich en español y si hay alguna diferencia semántica entre bocata, sándwich y bocadillo; y si el lema paella tiene un solo único tipo, por poner algunos ejemplos para ilustrar dicha cuestión. Es decir, el estudiante, delante de lo expuesto, se muestra insatisfecho con la limitación o, muchas veces, ausencia de información extralingüística por parte de algunas obras lexicográficas de esta categoría. Por esta razón, el aprendiz aspira a llegar más allá, o sea, él también anhela encontrar el componente cultural en la lectura del artículo, además de las informaciones propiamente lingüísticas $\mathrm{y}$, por supuesto, las definiciones y los ejemplos de uso disponen del suficiente espacio microestructural para ofrecer estas informaciones a sus consultantes.

A pesar de saber que la lengua y la cultura, brasileña e hispanoamericana, no presentan tantas 'diferencias y distancias' en su estructura, como ocurre entre la española y china, entre el español y marroquí, por ejemplo, es igualmente evidente que la incorporación de cuestiones culturales y/o enciclopédicas en el cuerpo del artículo de diccionario se convierte en un elemento facilitador más para la comprensión de la unidad léxica desconocida por el lector extranjero (ATIENZA CEREZO, 2005; MORENO; ATIENZA CEREZO, 2016; NADIN, 2017) a la hora de la búsqueda por la unidad léxica.

En este sentido, es importante destacar que el estudio sobre la utilización del diccionario en el aula todavía se encuentra en desarrollo (BATTANER, 2014); sin 
embargo, los problemas referentes a la elaboración de los artículos lexicográficos en dichas herramientas siguen vigentes, sobre todo los relacionados con las definiciones y los ejemplos de uso, puesto que las investigaciones más recientes como Celotti (2002) y Tungmen $\mathrm{Tu}$ (2004) entre otros, señalan que los contenidos de naturaleza enciclopédica y culturales, los centrados en la información sobre la realidad extralingüística de la unidad léxica descrita en el diccionario, no suelen estar contemplados en los repertorios, aunque sean relevantes para el desarrollo del aprendizaje de los aprendices extranjeros (TUNGMEN TU, 2004), e, incluso, no se registran en los paradigmas relativos al enunciado definicional y a los ejemplos de uso (GARRIGA ESCRIBANO, 2001; ROJAS ARREGOCÉS, 2016; MOREIRA, 2017).

Como ya hemos dicho anteriormente, se insiste nuevamente en señalar que consideramos que estas cuestiones subrayadas deben estar incluidas en la parte definitoria del lexema y en los ejemplos de uso, ya que se trata de una información enciclopédica y cultural que completa, añade y facilita la comprensión del destinatario y lector del diccionario, lo que inicialmente corrobora la idea del investigador Tungmen Tu (2004, p. 872) cuando declara:

En el caso de las palabras culturales, tenemos que dar a los usuarios la suficiente información para aprender a utilizar este tipo de palabras, de ahí que la información enciclopédica también deba ser incluida en la parte definitoria.

Por lo que sigue, este es un caso en el que vemos claramente que la información lingüística en el artículo lexicográfico no es del todo suficiente para que el consultante brasileño (estudiante, profesional y/o experto de un área específica), de cualquier nivel, comprenda efectivamente el significado real de un determinado lema, cuestión que abordaremos más detalladamente en el apartado de análisis de los datos de este estudio y como identificamos en nuestro trabajo doctoral en desarrollo (LIMA MOREIRA, en desarrollo). 
A propósito, es importante decir que el desarrollo del presente análisis tiene su base metodológica y teórica en los estudios recientes de Fuertes-Olivera y ArribasBaño (2008); Fuertes-Olivera (2009); Porto Dapena (2014) y Baccin (2017), y de trabajos anteriores de los teóricos Rey-Debove (1971), Seco (2003) y Lara (2006), entre otros, que investigan hace tiempo sobre la lexicografía pedagógica y su implicación en el aula, más específicamente, sobre las cuestiones referentes a la definición marcadas en los diccionarios de ELE, y sobre la presencia del contenido cultural en ellos, y, además, sobre cómo los ejemplos de uso son incorporados en dichos libros de referencia en esta ciencia, los cuales se consideran recursos lexicográficos positivos para el desarrollo del aprendizaje de una LE por varios estudios (JIMÉNEZ, 1986; RODRÍGUEZ BARCIA, 2016).

\section{Descripción del diccionario Señas y Diccionario de Español para Extranjeros estudiados en la investigación}

El Diccionario para la Enseñanza de la Lengua Española para Brasileños (SEÑAS, 2002) es una obra lexicográfica de significativa difusión y utilización en la enseñanza de ELE en Brasil (MOREIRA, 2017; SIMÃO, 2016), en diferentes niveles educacionales, tanto en las escuelas de enseñanza básica obligatoria y en los cursos libres de español, como en los grados universitarios de formación de los futuros profesores de ELE y también en las carreras en las que se imparten idiomas.

Es un diccionario que fue elaborado para fines de enseñanza de ELE para estudiantes brasileños como su propio título. Asimismo, cabe señalar que es del tipo bilingualizado o semibilingüe, ya que, al final de toda la información sobre la entrada descrita en lengua española, encontramos el equivalente del lema marcado en lengua portuguesa.

Por su parte, el Diccionario de Español para Extranjeros (2002) es una obra monolingüe dirigida a todos los estudiantes extranjeros, con definiciones claras de fácil comprensión. Además, ofrece ejemplos de uso en sus entradas. 


\section{Metodología}

Para la realización de nuestro estudio, analizamos las definiciones y los ejemplos de uso referentes a las entradas del ámbito del Turismo de las áreas de bebida, gastronomía y hostelería, dos de cada área, en total seis lemas, los cuales fueron elegidos del diccionario Señas y Diccionario de Español para Extranjeros.

En este sentido, este apartado del presente artículo está dedicado a describir los procedimientos metodológicos llevados a cabo para la extracción y selección de los términos analizados en esta investigación.

Los términos analizados fueron i) extraídos del libro de texto Nuevo Ven 1 (2007) adoptado en el curso de Turismo de una universidad pública de la esfera federal en Brasil, y ii) se comprobó si estos estaban recogidos en el repertorio Señas. La decisión de la elección de los términos se justifica porque, además de ser contemplados en el material didáctico utilizado por los estudiantes se considera que el léxico presente en el manual es relevante para el conocimiento de los estudiantes, profesionales en potencia, en cuanto aprendices de ELE y, sobre todo, en el momento de la ejecución de su labor profesional. A continuación, presentamos los términos seleccionados para este estudio:

Figura 1 -- Árbol de campo semántico del área de Turismo.

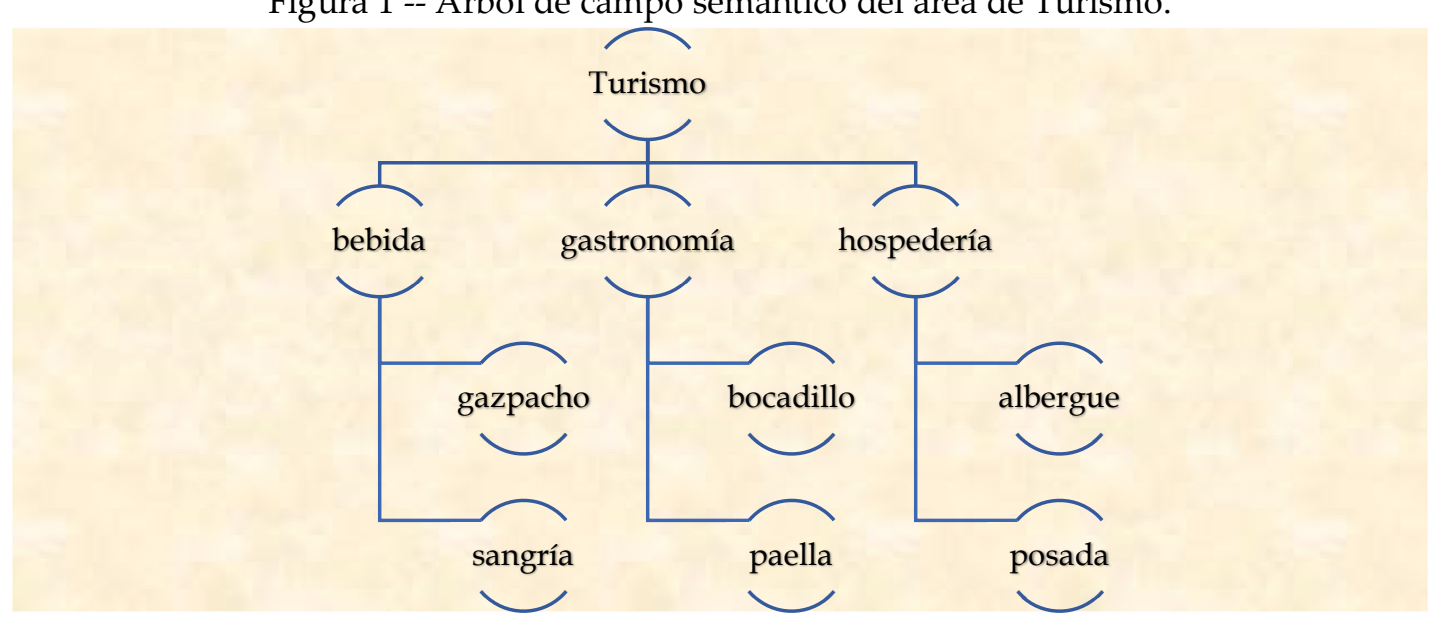


Una vez definido nuestro campo de interés para la realización del presente trabajo, desarrollamos el análisis propiamente dicho de las definiciones y de los ejemplos de uso a través de las informaciones aportadas por estos dos paradigmas lexicográficos encontradas en los seis artículos lexicográficos contemplados en la microestructura de los dos diccionarios. En la siguiente sección, presentamos el análisis y la discusión de los resultados recolectados.

\section{Análisis y discusión de los resultados recogidos}

Considerando lo anterior, presentamos el análisis descriptivo de las entradas seleccionadas, gazpacho y sangría, de la subrama de la bebida; los términos bocadillo y paella de la subrama de la gastronomía y, por último, los términos albergue y posada los cuales forman parte del campo léxico de la hospedería. Es importante señalar que, para este estudio, el análisis está limitado solamente a las definiciones y los ejemplos de uso, como hemos dicho antes, pero, aun así, los artículos están presentados en su integridad.

Así, como muestra de lo que hemos discutido anteriormente, empezamos nuestro análisis con los términos gazpacho y sangría, referentes al campo léxico bebida, que sirvieron de botón de muestra referente al tratamiento de las informaciones contempladas en cada artículo:

gaz.pa.cho IgaӨpátfo $\mid m$. Sopa fría formada por hortalizas, aceite, vinagre, sal y pan: el es una comida típica de Andalucía y Extremadura; para hacer hay que triturar bien todos los ingredientes; el $\sim$ se toma en el verano. $\square$ gaspacho

sangría I sangría| $\mathbf{1} f$. Bebida hecha con agua, *limonada, vino, azúcar y trozos de frutas: he puesto a la trozos de pera y de manzana; la se hace con vino tinto $y$ canela. $\rightarrow$ limonada. $\square$ sangria

Fuente: Señas, 2002. 
Nótese que los ejemplos anteriores de artículos de diccionarios muestran que la obra Señas, que es muy utilizada en los cursos de español en Brasil, parece no ser la más apropiada para los consultantes brasileños, puesto que este repertorio ofrece definiciones demasiado genéricas, no consistentes en descripciones y raramente extensas, ya que es una obra pensada para usuarios brasileños y la microestructura debe estar diseñada de esta manera, ofreciendo al lector de la herramienta pedagógica ayudas más allá de los lingüísticos. En este sentido, el usuario del diccionario no comprenderá de todo el significado del objeto lexicográfico en cuestión.

Tales artículos muestran que la definición es demasiado sintética, es decir, suprimen detalles pertinentes que facilitarían la comprensión lectora del artículo. De ahí que el hablante extranjero, en este caso el consultante brasileño, no pueda completar la información faltante por el registro restringido de la información

Sobre los ejemplos de uso, el primero usado en gazpacho no aporta ninguna información relevante al decir que los ingredientes son triturados, mientras que, en el segundo, presenta una información importante sobre cuándo se puede tomar esta bebida española. Sin embargo, debería decir que no solamente en el periodo mencionado, ya que dicha sopa fría está disponible en cualquier estación del año, a pesar de ser más común en verano, además de ser apreciada en todo el territorio español, aunque sea típica de las dos comunidades autónomas indicadas en la microestructura de diccionario.

Por tanto, vemos que el diccionario no explica suficientemente el significado de la palabra para que los lectores puedan tener subsidios lingüísticos más específicos y suficientes para sus producciones en lengua castellana, sean escritas $u$ orales, pues como afirma Castillo Carballo (2010, p. 1559): “Al fin y al cabo, el que aprende una lengua extranjera pretende adquirir una capacidad suficiente para confeccionar discursos que se adecuen a determinados patrones normativos". Por ello, esto pone de manifiesto que se requieren informaciones culturales y enciclopédicas para que el 
lector comprenda efectivamente el significado de estos alimentos, su uso y sus informaciones específicas sobre el lema.

En este sentido, continuamos nuestro análisis con los términos bocadillo y paella, relacionados con la subrama de la gastronomía:

bo.ca.di.llo s.m. 1. Trozo de pan cortado a lo largo en dos partes, y relleno con algún alimento: un bocadillo de chorizo. Dist. de sándwich (bocadillo con pan de molde). Se usa mucho la forma coloquial bocata.

pa.e.lla s.f. 1 Comida elaborada con arroz y con otros ingredientes, esp. carne, mariscos y legumbres: La paella es un plato de origen valenciano.

Fuente: Diccionario de Español para Extranjeros, 2002.

Como observamos, los paradigmas referentes a la definición y los ejemplos de uso no cumplen de todo la función y el objetivo que la microestructura de los diccionarios pedagógicos persigue desde el momento de su creación: definir y, sobre todo, aclarar el(los) empleo(s) de una palabra determinada, respectivamente. Sin embargo, el diccionario antes mencionado, Diccionario de Español para Extranjeros, de la editorial Ediciones SM, cumple adecuadamente su papel en cuanto obra didáctica y pedagógica en la definición de la entrada bocadillo.

Así pues, pensamos que en el lema anterior (bocadillo) las informaciones recogidas en la definición están seleccionadas y expuestas al lector brasileño de tal manera que podrán ser comprendidas por él claramente, sin la necesidad de mirar una fotografía, ir al país del idioma en cuestión o, incluso, mirar un vídeo en Internet para facilitar la comprensión del contenido de la definición lexicográfica, es decir, el lector entiende el verdadero significado de este artículo lexicográfico.

En lo tocante a los ejemplos de uso podemos observar que no se añade ningún tipo de información nueva como, por ejemplo, en bocadillo, el diccionario podría presentar ejemplos reales presentando los alimentos más típicos que suelen rellenar el bocadillo, y no únicamente uno como está marcado. Otro punto relevante es que el 
repertorio informa que bocadillo, sándwich y bocata se refieren a la misma realidad, pero son comidas distintas, aunque sean elaboradas con el principal alimento que es el pan.

Es probable que el usuario de Señas no utilice esta voz en su contexto de trabajo, pues en su microestructura ofrece una información insuficiente, e, incluso, una carencia de informaciones enciclopédicas las cuales puedan aclarar la definición de los artículos bocadillo y paella.

Relacionados con la subrama de la hostelería, destacamos los términos de referencia en esta área, albergue y posada, de acuerdo con el diccionario Señas (2002):

ha.bi.ta.ción laßita $\underline{\text { ión }}$ | $f$. Parte o pieza de una casa separada por paredes de las demás: tenemos que pintar esa porque las paredes están muy sucias. $\square$ cómodo

po.sa.da I posáðal $f$. Establecimiento situado en un camino que acoge a los viajeros: la diligencia hizo parada en una $\sim$. $\rightarrow$ mesón, venta. $\square$ hospedaria

Fuente: Señas, 2002.

Para la unidad léxica habitación, podemos decir que no se ha marcado ningún tipo de información más allá de la información más genérica de la palabra. Señas no presenta acepciones para que el consultante sepa que esta misma palabra se refiere también al espacio físico para hospedarse, además de que existen diferentes tipos. El ejemplo de uso está bien empleado, pero podría ser más específico para que el lector pueda fijar mejor el sentido de la palabra definida.

Como se puede observar en el artículo posada, el estudiante brasileño de ELE no va a comprender, adecuadamente, su significado exacto pues su definición no es clara ni precisa, lingüísticamente hablando, al leerla. Por ejemplo, el consultante no sabe si, para hospedarse, tendrá que efectuar algún pago. Además, no presenta la categoría, es decir, la forma mundial de indicar la calidad de los medios de hospedaje. En este artículo lexicográfico, las informaciones no favorecen la descodificación, ya que no 
facilitan una rápida comprensión exacta del artículo por la falta de otras informaciones más precisas, por ejemplo, informaciones enciclopédicas.

Es muy probable que el lector del diccionario no comprenda efectivamente el contenido semántico de los dos vocablos, pues no marca las informaciones enciclopédicas importantes al consultante brasileño y, sobre todo, a los usuarios del contexto de Turismo.

Para ejemplificar, presentamos el modelo de microestructura abstracta que defendemos ser ideal para un diccionario dirigido a la audiencia de estudiantes brasileños:

ca.ma.re.ro ra $\mid$ kamaréro, ra I m. $f$. 1 Profesional con o sin formación superior que se dedica a servir comidas o bebidas en diferentes sectores: Julio trabaja como camarero en un bar sirviendo a los clientes; La camarera que me atendió en el restaurant ha sido muy amable. 2 Profesional generalmente con studios universitarios responsible por la limpieza y organización de las habitaciones en un establecimiento hotelero: Señora, si necesitas toallas limpias, por favor, llame a la camarera de su piso; El camarero hará servicio de habitación cuando solicite a la recepción del hotel.

Como descrito en el cuadro anterior el artículo lexicográfico, la entrada será escrita en color rojo y con la marcación de la sílaba tónica en negrita y la separación silábica, seguida de la información fonética entre barras. Luego será presentada la información gramatical correspondiente a la palabra. Si la palabra tiene más de un significado, las acepciones serán marcadas con número en negrito para llamar la atención del lector y por orden de mayor frecuencia de uso. Las definiciones serán elaboradas con alguna información cultural y/o enciclopédica para facilita la comprensión del usuario. Es importante decir que para cada acepción, acreditamos que será, más de un ejemplo de uso, cuando posible y necesario, su empleo, que se convertirá en utilidad a la comprensión del consultante del diccionario. 


\section{Consideraciones finales}

Las voces de los investigadores Atienza Cerezo (2004), Salomão (2015), entre otros, destacan para la necesidad de presentar informaciones de tipo enciclopédicas y culturales en el cuerpo de los artículos lexicográficos, sobre todo, los dirigidos a la audiencia extranjera, pues discuten contenidos socioculturales a través de la relación entre cultura y sociedad, lo que constituyen informaciones extralingüísticas que ayudan a la formación del estudiante en un hablante más autónomo, capaz de interactuar con los nativos que tienen una manera diferente de pensar y ver el mundo.

Por esta razón, consideramos que el análisis de los paradigmas lexicográficos referentes a la definición y a los ejemplos de uso pueden ser diseñados a través de la inclusión de informaciones culturales y enciclopédicas, extralingüísticas, las cuales son de suma importancia para el conocimiento lingüístico y sociopragmático, conocimientos esenciales en el proceso de desarrollo de la enseñanza-aprendizaje del aprendiz extranjero.

El diccionario Señas, aunque haya sido elaborado con fines específicos y pedagógicos para los estudiantes brasileños, carece de una mayor atención en la incorporación de las informaciones que los responsables evalúan ser relevantes a este consultante. Por lo que consideramos que es fundamental para el proceso de aprendizaje y en el desarrollo de la competencia comunicativa de los aprendices de ELE y usuarios de diccionarios la incorporación de contenidos culturales en los diccionarios de aprendizaje para contribuir a la habilidad de poder comunicarse en otro código lingüístico (ATIENZA CEREZO, 2004; SALOMÃO, 2015).

Pensamos que el Diccionario de Español para Extranjeros (2002), repertorio lexicográfico elaborado con la pretensión de convertirse en una herramienta de apoyo para el aprendizaje de los consultantes no nativos y que, lamentablemente, no es muy utilizado en los contextos educativos brasileños, todavía requiere informaciones claras, es decir, bien definidas, en sus artículos lexicográficos relacionados con la 
temática analizada en este estudio. Creemos que eso se debe al desconocimiento de informaciones sobre las obras lexicográficas como, por ejemplo, al conocimiento de cómo elegir el diccionario adecuado, cómo y cuándo emplearlo, etc.

Pretendemos, también, contribuir efectivamente a los estudios acerca de la metalexicografía y a la lexicografía didáctica, en lo referente a la enseñanza del uso del diccionario en clase, buscando comprender cómo los diccionarios son utilizados por los profesores y aprendientes de ELE, saber si los artículos lexicográficos están bien definidos en tales diccionarios y si, por ejemplo, las informaciones en los artículos sirven al público de Turismo. Además, defendemos que este artículo contribuirá a la lexicografía aplicada, pues aspiramos a ofrecer modelos de entradas que recojan definiciones y ejemplos de uso con contenidos enciclopédicos y culturales.

\section{Referencias bibliográficas}

AHUMADA, I. Justificación lingüística de las insuficiencias que presentan los actuales diccionarios de la lengua. In: MOYA CORRAL, J. A. ; SOSINSKI, M (ed.). Lexicografía y enseñanza de la lengua española. Actas de las XI Jornadas sobre la enseñanza de la lengua española, Granada, 2006, p. 15-32.

ALVAR ESQUERRA, M. La enseñanza del léxico y el uso del diccionario. Madrid: Arco-Libros, 2003.

ATIENZA CEREZO. E. (2005). El tratamiento de la cultura en los diccionarios de aprendizaje. In: Revista electrónica de didáctica del español lengua extranjera (redELE); v. 2, n. 5, Madrid, p. 1-8.

ÁVILA MARTÍN, M. del C. El diccionario en el aula. Sobre los diccionarios escolares destinados a la enseñanza y aprendizaje del español como lengua extranjera. Granada: Edita: editorial. Universidad de Granada, 2000.

AZORÍN FERNÁNDEZ, D; SANTAMARÍA PÉREZ, M. I. In: DOMÍNGUEZ VÁZQUEZ, M. J.; SANMARCO BANDE, M. T. (ed.). Lexicografía y didáctica Diccionarios y otros recursos lexicográficos en el aula. Frankfurt: Peter Lang, 2017, p. 109-131. 
BACCIN, P. A função dos exemplos de uso em um dicionário pedagógico bilíngue voltado para a produção. In: NADIN, O.; ZAVAGLIA, C. (org.). Estudos do léxico em contextos bilíngues. Campinas: Editora Mercado de Letras, 2017. p. 97-113.

BATTANER, M. P. El léxico como pilar inicial de la reflexión lingüística y el diccionario. In: Anexos de Revista de Lexicografía, 32, Universidade da Coruña, A Coruña, 2014, p. 33-62.

BIDERMAN, M. T. C. Os dicionários na contemporaneidade: arquitetura, métodos e técnicas. In: ISQUERO, A. N.; OLIVEIRA, A. M. P. P. de. (org.) Ciências do Léxico. Lexicologia, Lexicografia, Terminologia da ANPOLL. Campo Grande: UFMS, 1998, p. 129.

BLECUA PERDICES, J. M. El diccionario como instrumento cultural de la lengua. In: SARIEGO LÓPEZ, I.; GUTIÉRREZ CUADRADO, J.; GARRIGA ESCRIBANO, C. (ed.). El diccionario en la encrucijada: de la sintaxis a la cultural digital. VII Congreso Internacional de Lexicografía Hispánica. Santander, Escuela Universitaria de Turismo Altamira, p. 51-66, 2017.

CASTILlO CARBALlO, M. A. Mecanismos de codificación en la producción lexicográfica monolingüe para la enseñanza del español. In: RUHSTALLER, S.; GORDÓN PERAL, M. D. Diccionario y aprendizaje del español. Investigación de Excelencia, 2010, p. 155-170.

CELOTTI, N. La culture dans les dictionnaires bilingues: où, comment, laquelle? In: Études de linguistique appliquée, 2002/4, 128, Université de Trieste, Trieste, p. 455456, 2002.

DOMÍNGUEZ VÁZQUEZ, M. J.; VALCÁRCEL RIVEIRO, C. Hábitos de uso de los diccionarios entre los estudiantes universitarios europeos: ¿nuevas tendencias? In: DOMÍNGUEZ VÁZQUEZ, M. J.; GÓMEZ GUINOVART, X.; VALCÁRCEL RIVEIRO, C. (org.): Lexicografía de las lenguas románicas II. Aproximaciones a la lexicografía contemporánea y contrastiva. Berlin: de Gruyter, 2015. p. 165-189.

FELIPE DE SOUZA, Á. M. El léxico en el discurso turístico en la enseñanza de E/LE: ¿Qué léxico enseñar y cómo enseñar? In: El diccionario como puente entre las lenguas y culturas del mundo. Actas del II Congreso Internacional de Lexicografía Hispánica. Alicante: Biblioteca Virtual Miguel de Cervantes, 2008. p. 458-466. Disponible en: http://www.cervantesvirtual.com/nd/ark:/59851/bmc417d9

FUERTES-OLIVERA, P. A.; ARRIBAS-BAÑO, A. Pedagogical Specialised Lexicography: The Representation of Meaning in English and Spanish Business 
Dictionaries. In: John Benjamins Publishing. Amsterdam/Philadelphia, John Benjamins, p. 289-302, 2008. DOI: https://doi.org/10.1075/tlrp.11

GARRIGA ESCRIBANO, C. Los ejemplos en los diccionarios didácticos del español. In: ANAYA CASTRO, M. (ed.). Diccionario y enseñanza. Alcalá: Universidad de Alcalá, 2001. p. 127-150.

HERNÁNDEZ HERNÁNDEZ, H. Los diccionarios de orientación escolar: contribución al estudio de la lexicografía monolingüe española. Tenerife: Universidad de la Laguna/Tübigen: Niemeyer, 1989. DOI: https://doi.org/10.1515/9783111340562

JIMÉNEZ JIMÉNEZ, B. Didáctica del vocabulario. Barcelona: Editorial Humanitas, 1986.

KRIEGER, M. da G. Dicionários para o ensino da língua materna: princípios e critérios de escolha. In: Revista Língua \& Literatura, v. 10 e 11, pp. 101-112, 2005.

LARA, L. F. El ejemplo en el artículo lexicográfico. In: BERNAL, E.; DECESARIS, J. (ed.). Palabra por palabra. Estudios ofrecidos a Paz Battaner. Barcelona: Universitat Pompeu Fabra, Institut Universitari de Lingüística Aplicada (Sèrie monografies, 10), 2006. p. 139-146.

LEHMANN, A.; MARTIN-BERTHET, F. Lexicologie, Sémantique, Morphologie, Lexicographie. Paris: Armand Colin, 2013.

LIMA MOREIRA, G. (2018, en desarrollo). Diccionario y enseñanza de ELE: propuesta de un modelo de artículo lexicográfico para estudiantes brasileños. (Tesis doctoral). Departamento de Traducción y Ciencias del Lenguaje, Universitat Pompeu Fabra.

MALDONADO GONZÁLEZ, C. Diccionarios y educación: ¿una relación de compromiso? In: NOMDEDEU RULL, A.; FORGAS BERDET, E.; BARGALLÓ ESCRIVÁ, M. (ed.). Avances de Lexicografía hispánica (I). Universitat Rovira i Virgili, 2012, p. 11-27.

MOREIRA, G. L. et al. Reflexões e ações no ensino e aprendizagem de Espanhol/LE. Fortaleza: EdUece, 2013.

MALDONADO, C.; NADIN, O. ¿Pero es qué todavía es útil usar el diccionario? Un diálogo sobre la importancia de su empleo en la clase de ELE con Concha Maldonado 
y Odair Nadin. In: Moreira, G. L. Revista Fórum Linguístico, v. 14, n. 3, p. 2388-2395. Universidade Federal de Santa Catarina, 2017.

MOREIRA, G. L. et al. Reflexões e ações no ensino e aprendizagem de Espanhol/LE. Fortaleza: EdUece, 2013.

MOREIRA, G. L. ¿Pero es qué todavía es útil usar el diccionario? Un diálogo sobre la importancia de su empleo en la clase de ELE con Concha Maldonado y Odair Nadin. In: MOREIRA, G. L. Revista Fórum Linguístico, v. 14, n. 3, Universidade Federal de Santa Catarina, p. 2388-2395, 2017. DOI: https://doi.org/10.5007/19848412.2017v14n3p2388

PONTES, A. L. Dicionário para uso escolar: o que é, e como se lê. Fortaleza: EdUece, 2009.

PRADO ARAGONÉS, J. El diccionario como recurso para la enseñanza del léxico: estrategias y actividad para su aprovechamiento. In: AYALA CASTRO, M. C. (coord.). Diccionarios y enseñanza. Universidad de Alcalá, pp. 205-226, 2001.

PORTO DAPENA, J. A. La Definición lexicográfica. Madrid: Arco/Libros, S. L., 2014.

REY-DEBOVE, J. Statut et fonction de l'exemple dans l'économie du dictionnaire. In: HEINZ, M. (ed.). L'exemple lexicographique dans les dictionnaires français contemporains. Paris: Lexicographica, 2005. p. 15-20. DOI: https://doi.org/10.1515/9783110924466.15

RODRÍGUEZ BARCIA, S. Introducción a la lexicografía. Proyecto editorial claves de la lingüística. Editorial Síntesis. Madrid, 2016.

ROJAS ARREGOCÉS, E. Los ejemplos en los diccionarios escolares de Colombia. Tesis doctoral. Programa de Traducción y Ciencias del Lenguaje. Universitat Pompeu Fabra, 2016.

SALOMÃO, A. C. B. O componente cultural no ensino e aprendizagem de línguas: desenvolvimento histórico e perspectivas na contemporaneidade. Trabalhos em Lingüística Aplicada, Campinas, N. 54.2. São Paulo, p. 361-392, 2015.

SANMARTÍN SAÉZ, J. In: MOREIRA, G. L. El diccionario de turismo como herramienta del aprendiz de ELE: entrevista con Julia Sanmartín Saéz. Caderno Virtual de Turismo. Rio de Janeiro, v. 17, n. 2., p. 5-8, 2017. 
SEÑAS. Diccionario para la enseñanza de la lengua española para brasileños. Universidad de Alcalá de Henares. 2ª ed. São Paulo: Martins Fontes, 2001.

SIMÃO, A. K. G. Unidades fraseológicas em dicionários bilíngues (EspanholPortuguês): más vale pájaro en mano que ciento volando. En NADIN, O.; ZAVAGLIA, C. (org.). Estudos do léxico em contextos bilíngues. Campinas: Editora Mercado de Letras, 2016. pp. 113-134.

SVENSÉN, B. A Handbook of Lexicography. The Theory and Practice of DictionaryMaking. Cambridge: Cambridge University Press, 2009.

TARP, S. Lexicography in the Borderland between Knowledge and NonKnowledge, Tübingen: Niemeyer, 2008. DOI: https://doi.org/10.1515/9783484970434

TORRE MADUEÑO, M. D. F. de la. Uso de los diccionarios y posibilidades pedagógicas en la enseñanza de segundas lenguas. El caso de filología inglesa. In: Diccionario y enseñanza. Universidad de Alcalá de Henares, 2001. p. 279-285.

TUNGMEN, Tu. La definición lexicográfica de los diccionarios bilingües españolchino en el aprendizaje de la cultura española. En ASELE, Actas XV, Sevilla, Universidad de Sevilla, pp. 868-874, 2004. 